Polymer Journal, Vol, 4, No. 1, pp 68-78 (1973)

\title{
Structural Studies of Poly(pentamethylene sulfide)
}

\author{
Yoshiharu Gotoh, Hideko Sakakihara, and Hiroyuki Tadokoro \\ Department of Polymer Science, Faculty of Science, Osaka University, \\ Toyonaka, Osaka, Japan.
}

(Received March 27, 1972)

\begin{abstract}
The crystal structure of poly(pentamethylene sulfide) $\left[-\left(\mathrm{CH}_{2}\right)_{5}-\mathrm{S}-\right]_{n}$, which is one of the polythioether series expressed by the general formula $\left[-\left(\mathrm{CH}_{2}\right)_{m}-\right.$ $\mathrm{S}-]_{n}$, has been studied by the use of X-ray diffraction and IR spectroscopic methods. The unit cell dimensions are $a=9.61 \AA, b=9.78 \AA, c$ (fiber axis) $=7.84 \AA$, and $\beta=131^{\circ}$, and the space group is $\mathbf{P} 2_{1} / \mathbf{a}\left(C_{2 h}^{5}\right)$. Four planar zigzag chains pass through the unit cell, and the fiber period consists of one monomeric unit.

The molecular packing in the lattice is different from both the polytetrahydrofurantype and the polyethylene-type in the polyether series $\left[-\left(\mathrm{CH}_{2}\right)_{m}-\mathrm{O}-\right]_{n}$. A pair of molecular chains become closer together with the $\mathrm{C}-\mathrm{S}-\mathrm{C}$ dipole moments antiparallel, and such pairs of molecules as a whole pack in the lattice just in the same way as for orthorhombic polyethylene. In the infrared spectra the progression bands and the crystal-field splitting arising from the $r\left(\mathrm{CH}_{2}\right)$ modes were observed. These infrared spectroscopic features were discussed on the basis of the simply coupled oscillator model.

KEY WORDS Poly(pentamethylene Sulfide) / Polythioethers / Polyethers / Planar Zigzag Conformation / Polytetrahydrofuran-Type Packing / Polyethylene-Type Packing / Progression Band / Crystal-Field Splitting /
\end{abstract}

In the previous papers, ${ }^{1-7}$ the structures of the polyether series expressed by the general formula $\left[-\left(\mathrm{CH}_{2}\right)_{m}-\mathrm{O}-\right]_{n}$ with $m=1-4,6,8-$ 10 , and 12 were determined by the use of X-ray diffraction and infrared spectroscopic methods. In the higher members of the polyethers $(m=4)$, the molecular structures take the essentially extended planar zigzag conformation, and two types of molecular packings (crystal forms) have been found; the monoclinic polytetrahydrofuran-type and the orthorhombic polyethylenetype. $^{2,5}$

In the polythioether series $\left[-\left(\mathrm{CH}_{2}\right)_{m}-\mathrm{S}-\right]_{n}$, the structure of polythiomethylene $(m=1)$ was determined by Carazzolo and Valle, ${ }^{8}$ and those of poly(ethylene sulfide $)^{9} \quad(m=2)$ and poly(trimethylene sulfide $)^{10}(m=3)$ were also determined by the authors. The structures of the higher members, however, have not yet been published so far. It is of considerable interest to examine whether the molecular and crystal structures of the higher members of polyethers are similar or not to the lower members of polyethers.

In this paper, the crystal structure of poly- (pentamethylene sulfide) $\left[-\left(\mathrm{CH}_{2}\right)_{5}-\mathrm{S}-\right]_{n}$ is studied by using mainly $\mathrm{X}$-ray diffraction method, and discussed in comparison with those of the higher members of the polyether series. Furthermore, as the infrared spectroscopic features attributed to the molecular and crystal structures, the band progression and the crystal-field splittings arising from the $r\left(\mathrm{CH}_{2}\right)$ modes are discussed in view of the phase difference relation between adjacent methylene groups.

\section{EXPERIMENTAL}

\section{Preparation of Samples}

Samples used in this study were synthesized by condensation polymerization of the disodium salt of 1,5-pentamethylenedithiol with 1,5-pentamethylenedibromide in absolute alcohol-dry benzene medium according to the procedure of Mavel and Kotch. ${ }^{11}$

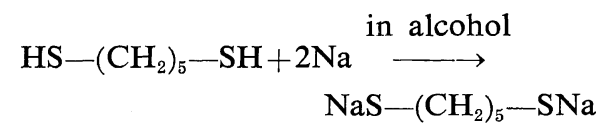




$$
\begin{aligned}
& \mathrm{NaS}-\left(\mathrm{CH}_{2}\right)_{5}-\mathrm{SNa}+\mathrm{Br}-\left(\mathrm{CH}_{2}\right)_{5}-\mathrm{Br} \\
& \underset{\text { reflux overnight }}{\longrightarrow}\left[-\left(\mathrm{CH}_{2}\right)_{5}-\mathrm{S}-\right]_{n}+\mathrm{NaBr}
\end{aligned}
$$

The fraction insoluble in the medium was separated, and washed with alcohol and with pure water until free from sodium bromide. It was dried under high vacuum and purified by precipitation from benzene solution using methanol as precipitant. In order to obtain a sample of high molecular weight, it was again fractionated with recrystallization from hot benzene solution.

The samples for X-ray measurements were prepared by quenching and stretching a molten sample in cold water (at about $10^{\circ} \mathrm{C}$ ), and annealing at $60^{\circ} \mathrm{C}$ overnight. The oriented film specimens for infrared measurements were prepared by rolling the powder sample at room temperature.

The melting point of this material is 73.0$74.5^{\circ} \mathrm{C}$. The measurement was made by the use of a polarizing microscope equipped with a hot stage. In Figure 1, the melting points for the polyether and polythioether series are plotted against the number of the methylene groups between oxygen or sulfur atoms. With the increase in this number, the melting points of both polymers at first decrease abruptly, and then increase gradually until they reach that of polyethylene. The melting point of poly(penta-

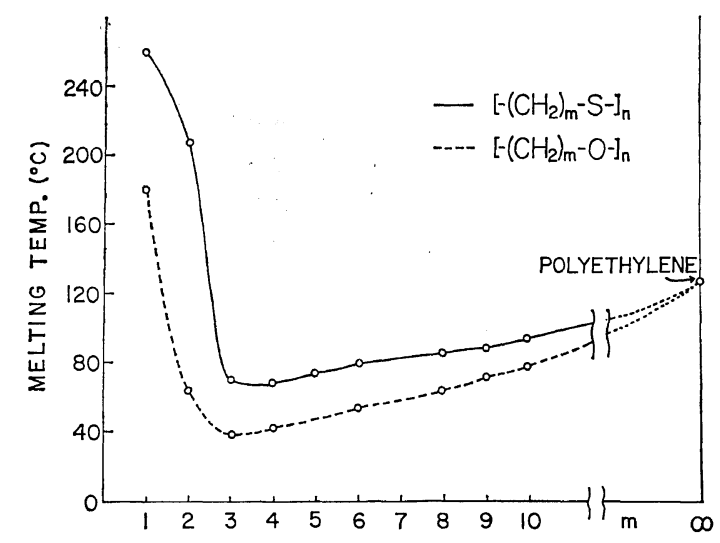

Figure 1. Melting temperatures for polyether and polythioether series plotted against the number of the methylene groups between the oxygen and sulfur atoms, respectively. methylene sulfide) is one of the lowest among the polythioether series.

\section{Measurements}

$\mathrm{X}$-ray diffraction measurements were made by using the cylindrical cameras (radii 50 and 35 $\mathrm{mm}$ ) with Ni-filtered $\mathrm{Cu}-\mathrm{K} \alpha$ radiation $(\lambda=1.542$ $\AA$ A). The intensities of the reflections obtained by the multiple film method were estimated by by visual comparison with a standard intensity scale. For the intensities thus obtained, some corrections were applied in the usual way. Forty-eight reflections were observed on the equatorial, first, second, and third layers. In Figure 2 is shown the fiber diagram of poly(pentamethylene sulfide).

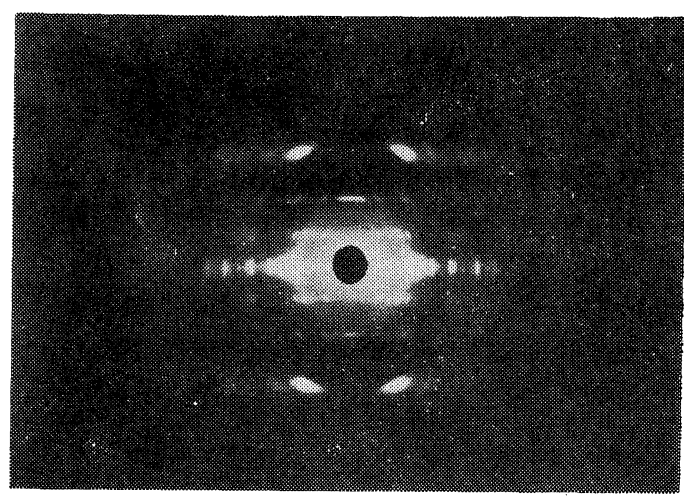

Figure 2. X-ray fiber photograph of poly(pentamethylene sulfide).

For the infrared absorption measurements, a Japan Spectroscopic Company DS-402G grating infrared spectrophotometer was used. The polarization measurements were made with a $\mathrm{AgCl}$ polarizer. In Figure 3, the polarized infrared spectra of the oriented polymer film are given in the region from 4000 to $700 \mathrm{~cm}^{-1}$. The solid and broken curves in the figure represent the absorptions obtained with polarized radiation with the electric vector perpendicular and parallel to the orientation direction, respectively.

\section{ANALYSIS}

\section{Unit Cell and Space Group}

All the observed reflections were indexed with a monoclinic unit cell, and the lattice constants are $a=9.61 \AA, b=9.78 \AA, c$ (fiber axis) $=7.84 \AA$, 


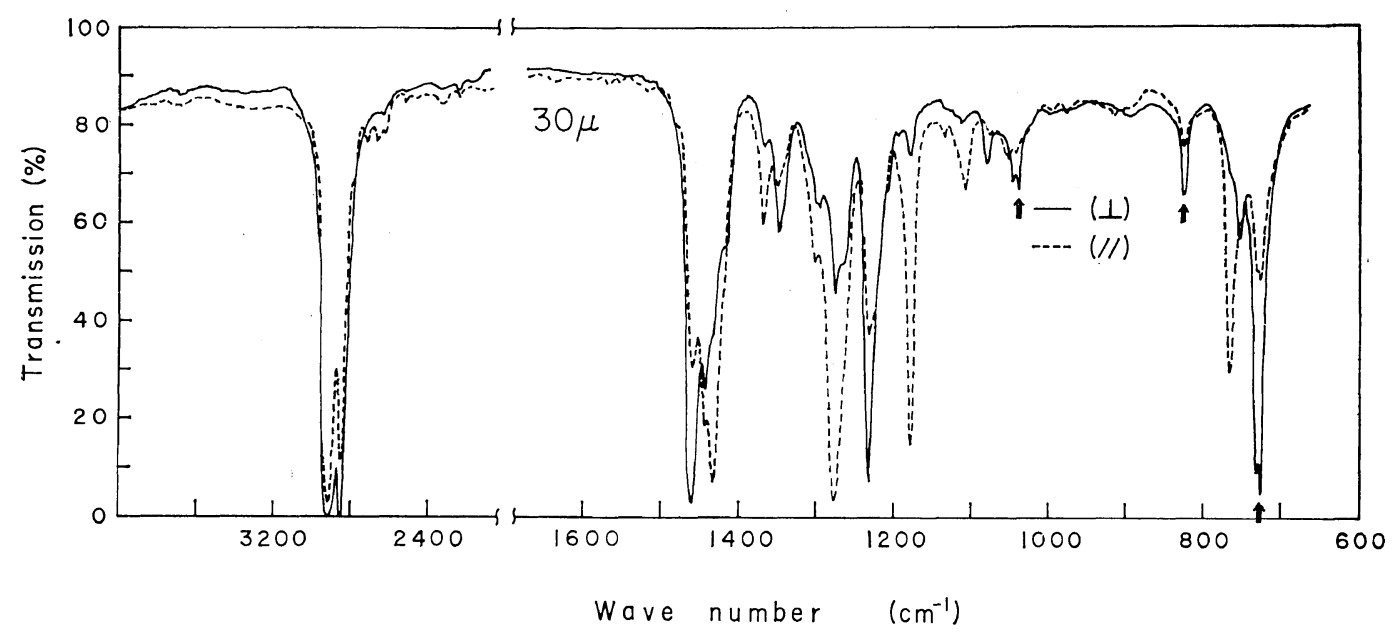

Figure 3. Polarized infrared absorption spectra of poly(pentamethylene sulfide). The solid (or broken) line represents the spectrum measured with the electric vector perpendicular (or parallel) to the orientation direction.

and $\beta=131^{\circ}$. Assuming that four monomeric units are contained in the unit cell, the calculated density leads to $1.213 \mathrm{~g} / \mathrm{cm}^{3}$. This value is in a satisfactory agreement with observed one, $1.132 \mathrm{~g} / \mathrm{cm}^{3}$, which was measured by the flotation method in aqueous $\mathrm{Na}_{2} \mathrm{~S}_{2} \mathrm{O}_{3}$ solution at $25^{\circ} \mathrm{C}$.

The observed systematic absences were $h=$ odd for $h 0 l$ reflections and $k=$ odd for $0 k 0$ reflections. This indicates that the space group is $\mathrm{P} 2_{1} / \mathrm{a}\left(\mathrm{C}_{2 \mathrm{~h}}^{5}\right)$.

\section{Molecular Conformation}

The possible molecular conformation of poly(pentamethylene sulfide) was considered to be an extended planar zigzag from the observed fiber period. If the molecular conformation is a planar zigzag, the fiber period consists of one monomeric unit $\left[-\left(\mathrm{CH}_{2}\right)_{5}-\mathrm{S}-\right]$, and the calculated fiber period becomes $7.98 \AA$ with the generally accepted bond lengths and bond angles $(\mathrm{C}-\mathrm{C}=1.54 \AA, \mathrm{C}-\mathrm{S}=1.81 \AA$, and all the bond angles are tetrahedral, $109^{\circ} 28^{\prime}$ ). The calculated value is slightly longer than the observed one, $7.84 \AA$, but this difference may be reasonable considering that the bond angle of $\mathbf{C}-\mathrm{S}-\mathrm{C}$ is generally smaller than the tetrahedral angle. For example, the $\mathrm{C}-\mathrm{S}-\mathrm{C}$ bond angles of dimethyl sulfide and DL-methionine are $105^{\circ}$ and $100^{\circ}$, respectively. ${ }^{12}$
It is also suggestive of the planar zigzag conformation that the average intensity of the third layer reflections, which correspond to the first layer line of polyethylene, is much stronger than those of the other layer lines as seen in Figure 2.

\section{Calculation of Structure Factors}

The calculation of the structure factors was made at first by assuming that the molecular chain takes an extended planar zigzag conformation with the following bond lengths and angles, $\mathrm{C}-\mathrm{C}=1.54 \AA, \mathrm{C}-\mathrm{S}=1.81 \AA, \angle \mathrm{CCC}=$ $109^{\circ} 28^{\prime}, \angle \mathrm{CSC}=100^{\circ}$, and $\angle \mathrm{CCS}=104^{\circ} 44^{\prime}$. Taking into account the aforementioned examples, the last two bond angles were estimated in such a way that the calculated fiber period coincided with the observed one. In the structure factor calculation, the contribution of the hydrogen atoms was taken into account. The coordinates were determined by assuming that the bond length $\mathrm{C}-\mathrm{H}$ and the bond angle $\mathrm{H}-\mathrm{C}-\mathrm{H}$ were $1.09 \AA$ and $109^{\circ} 28^{\prime}$, respectively, and that the $\mathrm{H}-\mathrm{C}-\mathrm{H}$ planes were perpendicular to the chain axis. Since the space group $P 2_{1} / a\left(C_{2 h}^{5}\right)$ has four equivalent general positions and four molecular chains pass through the unit cell, the molecular chains cannot be in the special positions. Applying the trial and error method to all the observed reflections, 
Table I. Atomic coordinates of poly(pentamethylene sulfide) $)^{\mathrm{a}}$

(an isotropic thermal factor, $B=10.0 \AA^{2}$ )

\begin{tabular}{cccc}
\hline Atom $^{\mathrm{b}}$ & $x / a$ & $y / b$ & $z / c$ \\
\hline $\mathrm{C}_{\mathrm{I}}$ & 0.262 & 0.091 & 0.722 \\
$\mathrm{C}_{\mathrm{II}}$ & 0.185 & 0.157 & 0.822 \\
$\mathrm{C}_{\mathrm{III}}$ & 0.262 & 0.091 & 0.046 \\
$\mathrm{C}_{\mathrm{IV}}$ & 0.185 & 0.157 & 0.146 \\
$\mathrm{C}_{\mathrm{V}}$ & 0.262 & 0.091 & 0.370 \\
$\mathrm{~S}$ & 0.157 & 0.180 & 0.462 \\
\hline
\end{tabular}

a Atomic coordinates of the hydrogen atoms are omitted.

$\mathrm{b}$ The atoms are numbered as

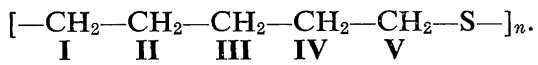

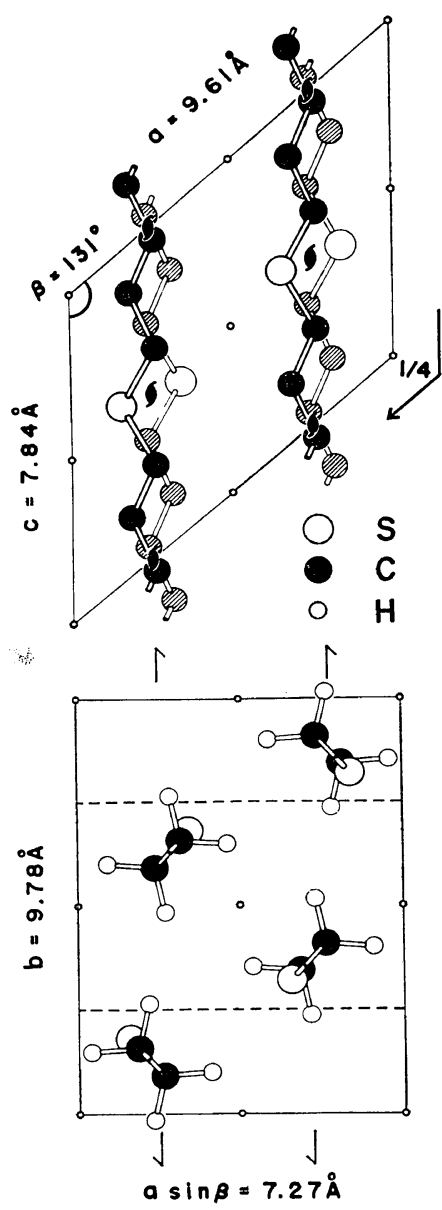

Figure 4. Crystal structure of poly(pentamethylene sulfide). the resulting discrepancy factor

$\boldsymbol{R}\left(\boldsymbol{R}=\Sigma|| \boldsymbol{F}_{\mathrm{o}}|-| \boldsymbol{F}_{\mathrm{c}} \| / \Sigma\left|\boldsymbol{F}_{\mathrm{o}}\right|\right)$ was $15 \%$.

Starting from this result, the atomic coordinates were refined by the least squares method. Here, the full matrix least squares method based on the internal coordinates ${ }^{13,14}$ was applied, since the number of the parameters is too large compared with the number of the observed reflections to make use of the ordinary method. Then, the number of variables can be reduced by fixing the values of the bond lengths, bond angles, and torsional angles, and the positions of the molecular chains and the value of the temperature factor were refined. The calculation was made by using a program prepared by Mr. Yasuhiro Takahashi of this laboratory. ${ }^{14}$ For the bond lengths and bond angles, the standard values aforementioned were used at first, and were finally corrected to the following values, $\mathrm{C}-\mathrm{C}=1.533 \AA^{*}, \mathrm{C}-\mathrm{S}=1.80 \AA$, $\mathrm{C}-\mathrm{H}=1.07 \AA^{*}, \angle \mathrm{CCC}=112^{\circ} *, \angle \mathrm{CCS}=106^{\circ}$, and $\angle \mathrm{HCH}=107^{\circ *}$. The values marked with asterisks were cited from the result of the structure analysis of hexatriacontane $\mathrm{C}_{36} \mathrm{H}_{74}$ reported by Teare. ${ }^{15}$

After ten cycles, the discrepancy factor R was reduced to $8 \%$ and the isotropic thermal factor $B$ became $10.0 \AA^{2}$ for all the atoms. The final atomic coordinates are listed in Table I. The final crystal structure is shown in Figure 4. The comparisons between the observed and calculated structure factors are given in Table II.

\section{RESULT AND DISCUSSION}

\section{Crystal Structure}

It has been found for the higher members of the polyether series $\left[-\left(\mathrm{CH}_{2}\right)_{m}-\mathrm{O}-\right]_{n} .(m=4,6$, $8-10$, and 12) that the molecules take the essentially extended planar zigzag conformation and that there are two types of molecular packings, the monoclinic polytetrahydrofuran type (PTHF-type) and the orthorhombic polyethylenetype (PE-type). ${ }^{5}$ In Figure 5 are shown the molecular packings ( $c$ projection) of (a) PTHFtype and (b) PE-type. Van der Waals radii of the chain atoms are indicated by circles. In these two types, two essentially planar zigzag chains pass through the center and the corner 
Y. Gotoh, H. SAKakihara, and H. TADOKoro

Table II. Observed and calculated structure factors for poly(pentamethylene sulfide)

\begin{tabular}{|c|c|c|c|c|c|}
\hline$h k l$ & $\sqrt{\bar{I}_{0}}{ }^{a}$ & $\sqrt{I_{\mathrm{c}^{\mathrm{b}}}^{\mathrm{b}}}$ & $h k l$ & $\sqrt{\bar{I}_{0}}{ }^{\mathrm{a}}$ & $\sqrt{I_{\mathrm{c}}}$ \\
\hline 110 & 40.9 & 31.5 & $\overline{2} 41$ & - & 1.3 \\
\hline 020 & 30.5 & 29.1 & $\overline{2} 02$ & 19.1 & 16.7 \\
\hline 120 & 216.1 & 210.8 & $\overline{2} 12$ & 24.8 & 21.5 \\
\hline 200 & 120.0 & 122.6 & $\overline{1} 12$ & - & 1.1 \\
\hline $\begin{array}{lll}2 & 1 & 0\end{array}$ & $\begin{array}{l}90.9 \\
58.7\end{array}$ & 96.3 & $\left(\begin{array}{lll}0 & 0 & 2 \\
2 & 2 & 2\end{array}\right)$ & & \\
\hline $\begin{array}{lll}1 & 3 & 0 \\
2 & 2 & 0\end{array}$ & $\begin{array}{c}58.7 \\
-\end{array}$ & $\begin{array}{r}60.1 \\
1.2\end{array}$ & $\left.\begin{array}{lll}2 & 2 & 2 \\
1 & 2 & 2\end{array}\right\}$ & 34.3 & 36.8 \\
\hline $\begin{array}{lll}0 & 4 & 0\end{array}$ & & $\begin{array}{r}1.2 \\
57.8\end{array}$ & $\left.\begin{array}{llll}\frac{1}{3} & 1 & 2\end{array}\right)$ & & \\
\hline $\begin{array}{lll}2 & 3 & 0\end{array}$ & 58.5 & 51.8 & 012 & 22.1 & 18.0 \\
\hline $\left.\begin{array}{lll}3 & 1 & 0 \\
1\end{array}\right\}$ & 34.0 & 42.0 & $\overline{3} 22$ & - & 2.9 \\
\hline $\begin{array}{lll}14 & 0\end{array}$ & 34.0 & 42.0 & 022 & 20.4 & 19.5 \\
\hline 320 & 31.7 & 34.9 & $\overline{2} 32$ & - & 8.7 \\
\hline 240 & 30.7 & 31.8 & 132 & - & 2.3 \\
\hline 330 & 52.7 & 57.2 & $\overline{4} 02$ & $\mathrm{w}^{\mathrm{c}}$ & 10.5 \\
\hline 150 & 20.5 & 25.4 & $\overline{4} 12$ & 24.7 & 24.1 \\
\hline $\begin{array}{lll}4 & 1 & 0 \\
2 & 5 & 0\end{array}$ & 32.4 & 36.5 & $\left.\begin{array}{lll}\overline{3} & 3 & 2 \\
1 & 1 & 2\end{array}\right\}$ & 25.3 & 27.0 \\
\hline $\begin{array}{lll}3 & 4 & 0 \\
4 & 2 & 0\end{array}$ & 41.9 & 45.6 & 032 & - & 3.7 \\
\hline $\begin{array}{llll}4 & 2 & 0 \\
0 & 6 & 0\end{array}$ & 9.0 & 11.9 & $\overline{4} 22$ & - & 6.0 \\
\hline 160 & - & 3.2 & $\left.\begin{array}{lll}\overline{2} & 4 & 2 \\
1 & 4 & 2\end{array}\right\}$ & & \\
\hline 430 & - & 9.1 & $\begin{array}{lll}1 & 2 & 2\end{array}$ & 27.0 & 26.7 \\
\hline 350 & 31.0 & 26.8 & $\left.\begin{array}{lll}\overline{1} & 1 & 3\end{array}\right\}$ & 58.0 & 56.8 \\
\hline 260 & - & 1.4 & $\left.\begin{array}{lll}\overline{2} & 2 & 3\end{array}\right\}$ & 58.0 & 56.8 \\
\hline $\begin{array}{llll}0 & 0 & 1\end{array}$ & 22.7 & 24.4 & $\left.\begin{array}{ccc}4 & 0 & 3 \\
3 & 2 & 3\end{array}\right\}$ & 28.1 & 23.1 \\
\hline$\overline{1} 11$ & - & 4.3 & 413 & 50.8 & 46.8 \\
\hline 011 & 12.7 & 11.6 & 123 & 27.3 & 27.6 \\
\hline$\overline{2} 01$ & 13.3 & 12.1 & $\left.\begin{array}{lll}\overline{2} & 3 & 3\end{array}\right\}$ & 26.7 & 27.6 \\
\hline$\overline{2} 11$ & 23.9 & 30.5 & $\begin{array}{l}\overline{4} 23 \\
-1\end{array}$ & 20.7 & 27.6 \\
\hline$\overline{1} 21$ & 22.2 & 21.7 & $\left.\begin{array}{lll}\overline{3} & 3 & 3 \\
0 & 0 & 3\end{array}\right\}$ & 46.1 & 44.7 \\
\hline 021 & 24.8 & 27.3 & $\begin{array}{lll}0 & 13\end{array}$ & 33.7 & 32.8 \\
\hline $\left.\begin{array}{lll}1 & 1 & 1 \\
2 & 2 & 1\end{array}\right\}$ & 20.1 & 19.1 & 133 & - & 5.7 \\
\hline $\begin{array}{lll}2 & 1 & 1\end{array}$ & $\mathrm{w}^{\mathrm{c}}$ & 10.6 & $\left.\begin{array}{lll}0 & 2 & 3\end{array}\right)$ & & \\
\hline $\left.\begin{array}{lll}\frac{1}{3} & 2 & 1 \\
1\end{array}\right\}$ & 13.6 & 18.2 & $\left.\begin{array}{ccc}\overline{5} & 1 & 3 \\
& 3 & 3\end{array}\right\}$ & 31.7 & 32.0 \\
\hline $\begin{array}{lll}3 & 1 & 1 \\
0 & 3 & 1\end{array}$ & $\begin{array}{r}13.0 \\
-\end{array}$ & $\begin{array}{r}10.2 \\
3.4\end{array}$ & $\left.\begin{array}{lll}\overline{3} & 4 & 3\end{array}\right)$ & & \\
\hline $\begin{array}{lll}\overline{2} 31 & 1\end{array}$ & $\overline{\mathrm{w}^{\mathrm{c}}}$ & $\begin{array}{r}3.4 \\
11.1\end{array}$ & $\begin{array}{l}\overline{2} \\
\overline{5}\end{array} 43$ & 44.2 & 43.7 \\
\hline$\overline{3} 21$ & - & 2.8 & $\begin{array}{lll}\overline{5} & 2 & 3 \\
0 & 3 & 3\end{array}$ & & \\
\hline 131 & 20.9 & 21.2 & $\left.\begin{array}{lll}0 & 3 & 3 \\
1 & 4 & 3\end{array}\right\}$ & 46.1 & 40.3 \\
\hline 211 & 22.3 & 27.3 & $\left(\begin{array}{lll}1 & 1 & 3 \\
4\end{array}\right)$ & & \\
\hline$\overline{1} 41$ & 22.7 & 28.0 & 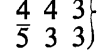 & 36.0 & 36.5 \\
\hline 041 & - & 1.9 & & & \\
\hline$\overline{3} 31$ & 17.3 & 25.1 & $\left.\begin{array}{lll}12 & 2\end{array}\right\}$ & 31.5 & 26.3 \\
\hline
\end{tabular}

a The observed structure factors $\sqrt{I_{\mathrm{o}}}$ were put on the same scale as the $\sqrt{I_{\mathrm{c}}}\left(=\sqrt{m F_{\mathrm{c}}^{2}}\right)$ by setting $\Sigma k \sqrt{I_{0}}=\Sigma \sqrt{m F_{\mathrm{c}}^{2}}$, where $k$ is the scale factor and $m$ is the multiplicity.

b $\sqrt{I_{\mathrm{c}}}$ of the reflections which overlap on X-ray fiber photographs are $\sqrt{\Sigma m F_{\mathrm{c}}}$.

c w, weak. 


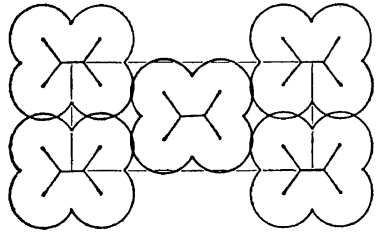

(a)

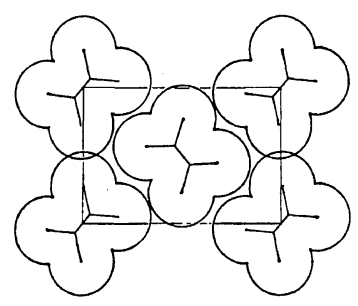

(b)

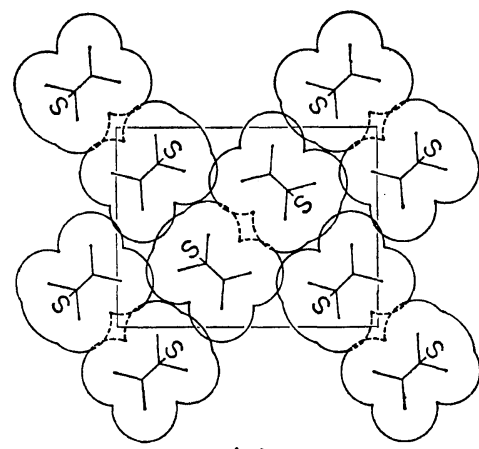

(c)

Figure 5. Molecular packings (c-axis projections) of (a) polytetrahydrofuran-type, (b) polyethylenetype, and (c) poly(pentamethylene sulfide). Van der Waals radii of the chain atoms are indicated by the circles.

of the unt cell, but the molecular arrangements are different in the two types; the zigzag planes of the molecular chains are parallel to the $b c$ plane in the PTHF-type, while the zigzag planes make an angle of about $41^{\circ}$ with $b c$ plane in the PE-type.

The PTHF-type is generally more stable in the case of the lower $m$ members $(m=4,6$, and 8 ), and the PE-type is stable in the case of the higher $m$ members ( $m=8-10$ and 12), though the occurrence of the crystal modifications depends on the preparation conditions ${ }^{5}$ (from melt or solution). The stability of these crystal structures is generally attributed to the electrostatic and van der Waals interactions. It may be concluded qualitatively from the experimental results that the crystal structures of these polyethers are produced by the stronger effects of the dipole-dipole interaction between the $\mathrm{C}-\mathrm{O}-\mathrm{C}$ groups in the PTHF-type and the van der Waals interaction between the methylene sequences in the PE-type.

At first, it was expected from the chemical structure that the crystal structure of poly(pentamethylene sulfide) would also be similar to one of the two types of polyethers. In the present work, however, it has been found that the crystal structure is different from both types as seen in Figure 5. Since the polyethers abovementioned are mainly $m=$ even, the oxygen atoms in the molecular chain are located alternately on the both sides of the zigzag chain, and so the antiparallel array of the $\mathrm{C}-\mathrm{O}-\mathrm{C}$

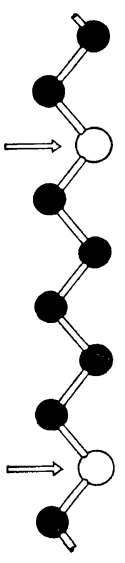

(a)

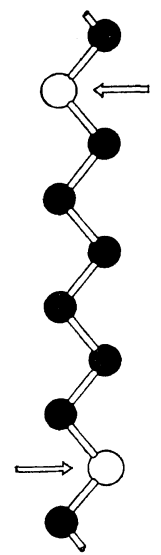

(b)
Figure 6. Arrangements of the chain atoms and the directions of dipole moments: (a) $m=$ odd; (b) $m=$ even.

dipole moments contributes to the stabilization of the planar zigzag conformation (Figure $6 \mathrm{~b}$ ). On the other hand, in poly(pentamethylene sulfide), all the sulfur atoms are located on one side of the zigzag chain because of $m=$ odd, and the $\mathrm{C}-\mathrm{S}-\mathrm{C}$ dipole moments are set in a parallel array to each other (Figure 6a). Therefore, the planar zigzag conformation of poly(pentamethylene sulfide) may be unstable in an isolated molecular chain, but stabilized as a whole by the arrangement of the two molecular chains whereby the zigzag planes are parallel 
and the dipole moments are antiparallel to each other.

Poly(oxacyclobutane $)\left[-\left(\mathrm{CH}_{2}\right)_{3}-\mathrm{O}-\right]_{n}$ is a good example that the planar zigzag conformation is unstable in an isolated chain for the parallel array of the $\mathrm{C}-\mathrm{O}-\mathrm{C}$ dipole moments. In this polyether, three crystal modifications have been found, ${ }^{3}$ and one of those is stable only in the presence of water as a crystal hydrate. In this case, the planar zigzag conformation may be stabilized by the formation of hydrogen bonds with water molecules between the molecular chains. In poly(nonamethylene oxide $)^{5}\left[-\left(\mathrm{CH}_{2}\right)_{9}\right.$ $-\mathrm{O}-]_{n}$, however, the PE type is stable in spite of $m=$ odd. In this case, the number $m$ is so large that the crystal structure may be independent of whether $m=$ odd or even because of the stronger effect of the van der Waals interaction between the long methylene sequences.

The relation of the two neighboring poly(pentamethylene sulfide) chains arranged in parallel is shown in Figure 7a. It can be clearly seen from this figure that each chain atom of one molecular chain is located in the position between two chain atoms of the other molecular chain so that these chains can be efficiently packed. This relation is similar to that of the two neighboring molecular chains arranged in parallel in polytetrahydrofuran as shown in Figure $7 \mathrm{~b}$. The distance between the two

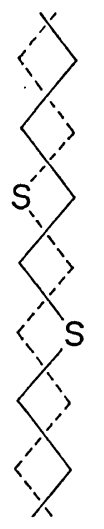

(a)

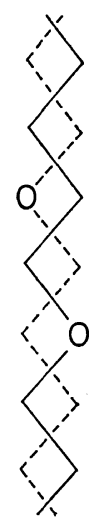

(b)
Figure 7. Arrangements of the two neighboring molecules parallel to each other viewed perpendicular to the molecular planes: (a) poly(pentamethylene sulfide); (b) polytetrahydrofuran. neighboring molecular planes in poly(pentamethylene sulfide), $4.0 \AA$, is very close to the value in polytetrahydrofuran, $3.9 \AA$. If these paired chains can be regarded as forming a united molecular chain, the molecular packing of poly(pentamethylene sulfide) crystal is quite similar to that of the PE-type as can be seen in Figures 5b and 5c. It may be concluded, therefore, that the two molecular chains are arranged in parallel to each other like those of the PTHF-type due to the dipole-dipole interactions and are packed as a whole like the PE-type due to the van der Waals interactions.

It is to be expected from the above consideration that the crystal structures of the other polythioethers with $m=$ even will be similar to either of the two types in the polyether series. This will be definitely confirmed by the structure analyses of poly(hexamethylene sulfide) $\left[-\left(\mathrm{CH}_{2}\right)_{6}-\mathrm{S}-\right]_{n}$, etc., which are now being carried out by the authors. On the other hand, it is also to be expected that the crystal structure of poly(heptamethylene oxide) $\left[-\left(\mathrm{CH}_{2}\right)_{7}\right.$ $-\mathrm{O}-]_{n}$ will be similar to that of poly(pentamethylene sulfide). The structure analysis is now in progress, and the results at the present stage are in accordance with expectations. ${ }^{16}$ However, poly(pentamethylene oxide) $\left[-\left(\mathrm{CH}_{2}\right)_{5}\right.$ $-\mathrm{O}-]_{n}$ having the identical number $m$ with poly(pentamethylene sulfide) has not yet been synthesized because of the predominant ring closure in the condensation polymerization.

\section{Infrared Spectra and the Structure}

The infrared spectroscopic features of poly(pentamethylene sulfide) are well explained by the molecular conformation and the crystal structure as determined by X-ray analysis. An infinitely extended planar chain of this molecule has three kinds of symmetry elements as shown

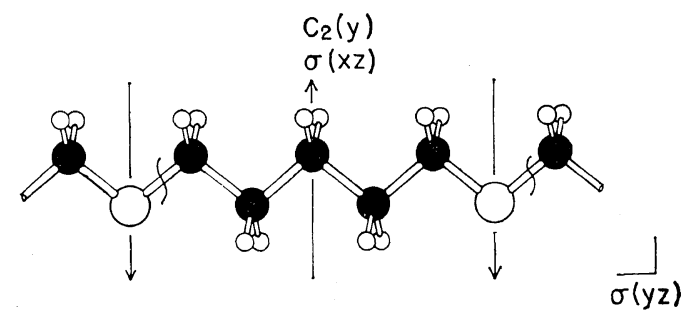

Figure 8. Symmetry elements of a poly(pentamethylene sulfide) chain. 
Table III. Character table, numbers of normal modes, and selection rules for poly(pentamethylene sulfide)

\begin{tabular}{ccccccc}
\hline $\mathrm{C}_{2 \mathrm{~V}}$ & $\mathrm{E}$ & $\mathrm{C}_{2}(y)$ & $\sigma(y z)$ & $\sigma(x y)$ & $N^{\mathrm{a}}$ & Infrared $^{\mathrm{b}}$ \\
\hline $\mathrm{A}_{1}$ & 1 & 1 & 1 & 1 & $14-\mathrm{T}_{y}$ & $\mathrm{~A}(\perp)$ \\
$\mathrm{A}_{2}$ & 1 & 1 & -1 & -1 & 9 & $\mathrm{~F}$ \\
$\mathrm{~B}_{1}$ & 1 & -1 & 1 & -1 & $13-\mathrm{T}_{x}$ & $\mathrm{~A}(\|)$ \\
$\mathrm{B}_{2}$ & 1 & -1 & -1 & 1 & $12-\mathrm{T}_{x}, \mathrm{R}_{z}$ & $\mathrm{~A}(\perp)$ \\
\hline
\end{tabular}

a $N$, number of total normal modes under each symmetry species; $\mathrm{T}_{x}, \mathrm{~T}_{y}$, and $\mathrm{T}_{z}$, pure translations parallel to each direction; $\mathrm{R}_{z}$, pure rotation about the chain axis.

b A, active; F, forbidden.

in Figure 8; the two-fold rotation axes coinciding with the bisectors of the angles $\mathrm{C}-\mathrm{S}-\mathrm{C}$ and $\mathrm{C}-\mathrm{C}-\mathrm{C}$, the mirror plane coinciding with the molecular plane, and the mirror planes containing the two-fold rotation axes and perpendicular to the molecular plane. The molecular group is therefore isomorphous to the point group $\mathrm{C}_{2 \mathrm{v}}$. In Table III are given the the symmetry species, character table, numbers of normal modes, and selection rules obtained from the factor group analysis. Here, $N$ is the number of normal modes in each symmetry species. $\mathrm{T}_{x}, \mathrm{~T}_{y}$, and $\mathrm{T}_{z}$ are pure translations parallel to each direction and $\mathbf{R}_{z}$ is pure rotation about the chain axis.

In the planar zigzag polyethers and polythioethers, in general the number of the individual $\mathrm{CH}_{2}$ group modes such as stretching, rocking, etc. depends systematically upon the number of the methylene groups involved in the fiber period. An approximate vibrational analysis ${ }^{17}$ of these progressions based on a simply coupled oscillator model provides experimentally the frequency-phase difference relations. In this approximate treatment, the vibrational mode of each individual $\mathrm{CH}_{2}$ group is replaced by a harmonic oscillator with the frequency characteristic of the mode under consideration, and then the oxygen or sulfur atoms can be regarded as the fixed end. ${ }^{7}$ The vibrational frequency $\nu_{k}$ of a linear array of $m$ identical oscillators depends on the phase difference $\delta_{k}$ between adjacent oscillators. The phase difference allowed for the system is expressed by

$$
\delta_{k}=k \pi /(m+1) \quad(k=1,2, \ldots, m)
$$

Table IV. Relation between $k$-value and symmetry species for poly(pentamethylene sulfide)

\begin{tabular}{lcc}
\hline \multirow{2}{*}{ Mode $^{\mathrm{a}}$} & \multicolumn{2}{c}{ Factor group $\mathrm{C}_{2 \mathrm{~V}}$} \\
\cline { 2 - 3 } & $k=$ odd & $k=$ even \\
\hline $\mathrm{CH}_{2}$ symmetric stretching & $\mathrm{A}_{1}$ & $\mathrm{~B}_{1}$ \\
$\mathrm{CH}_{2}$ antisymmetric stretching & $\mathrm{B}_{2}$ & $\mathrm{~A}_{2}$ \\
$\mathrm{CH}_{2}$ bending & $\mathrm{A}_{1}$ & $\mathrm{~B}_{1}$ \\
$\mathrm{CH}_{2}$ wagging & $\mathrm{B}_{1}$ & $\mathrm{~A}_{1}$ \\
$\mathrm{CH}_{2}$ twisting & $\mathrm{A}_{2}$ & $\mathrm{~B}_{2}$ \\
$\mathrm{CH}_{2}$ rocking & $\mathrm{B}_{2}$ & $\mathrm{~A}_{2}$ \\
\hline
\end{tabular}

a Only the $\mathrm{CH}_{2}$ group modes are listed.

Assignments of integers $k$ to the observed bands can be made on the basis of the selection rules which are expressed in terms of the value of $k$ and the number of methylene groups $m$ for each vibrational mode. The results for poly(pentamethylene sulfide), $m=5$, are given in Table IV. Of the symmetry species in the table, $A_{1}, B_{1}$, and $B_{2}$ are infrared-active.

The $\mathrm{CH}_{2}$ rocking modes $r\left(\mathrm{CH}_{2}\right)$ are discussed below, since the spectroscopic features of these modes reflect especially the molecular

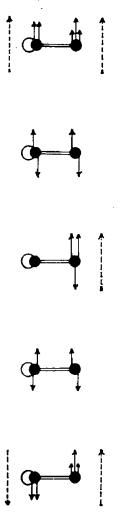

(a)
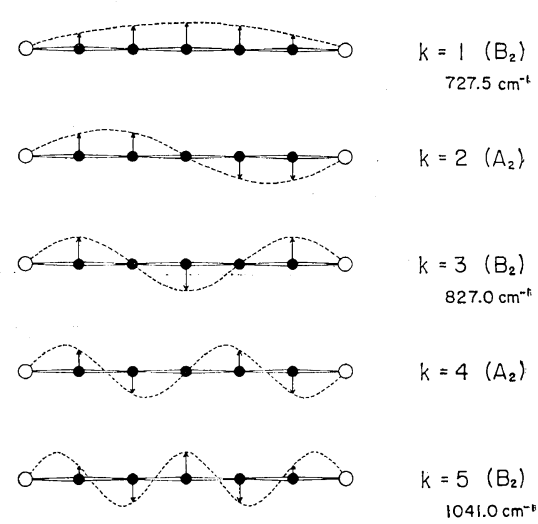

(b)
Figure 9. Schematic representations of the $r\left(\mathrm{CH}_{2}\right)$ modes for each $k$-value viewed parallel to (a) the chain axis and (b) the molecular plane in poly(pentamethylene sulfide). The arrows drawn with the solid and broken lines indicate the direction of the displacement of the hydrogen atoms from the molecular plane and the direction of the total transition moments associated with the $\mathrm{CH}_{2}$ groups in each side of the zigzag chain, respectively. 
and crystal structures of poly(pentamethylene sulfide); the $r\left(\mathrm{CH}_{2}\right)$ modes are actually observed as the progression bands characteristic of the methylene sequences, and the behavior of the absorption intensities and the dichroisms can be clearly explained by means of the factor group analysis. Five $r\left(\mathrm{CH}_{2}\right)$ modes $\left(\mathrm{A}_{2}\right.$ and $\left.\mathrm{B}_{2}\right)$ can be considered from the frequency-phase difference relation, and the three modes with $k=$ odd $\left(\mathbf{B}_{2}\right)$ are infrared-active. These five modes are schematically shown in Figure 9, in which the arrows indicate the directions of the displacements of the hydrogen atoms from the molecular plane. The bands arising primarily from $r\left(\mathrm{CH}_{2}\right)$ modes are observed mainly in the region from 1050 to $700 \mathrm{~cm}^{-1}$. The perpendicular bands shown with the arrows in Figure 3 correspond to the three modes, $727\left(\delta_{1}=\pi / 6\right)$, $827\left(\delta_{3}=3 \pi / 6\right)$, and $1041 \mathrm{~cm}^{-1} \quad\left(\delta_{5}=5 \pi / 6\right)$. As shown in Figure 10, the frequencies of the observed progression bands correspond approximately to a point of the dispersion curve of the $r\left(\mathrm{CH}_{2}\right)$ modes calculated for planar zigzag polymethylene by Tasumi, et al. ${ }^{18}$

The doublets (the splitting width $\Delta \nu$ about $5 \mathrm{~cm}^{-1}$ ) observed at about 727 and $1041 \mathrm{~cm}^{-1}$ are considered to be due to the band splittings of the $\mathrm{CH}_{2}$ rocking vibrations caused by the intermolecular interaction between the hydrogen atoms in the crystal, as generally known in orthorhombic polyethylene. ${ }^{19}$ The space group of poly(pentamethylene sulfide) is $\mathrm{P} 2_{1} / \mathrm{a}\left(\mathrm{C}_{2 \mathrm{~h}}^{5}\right)$,

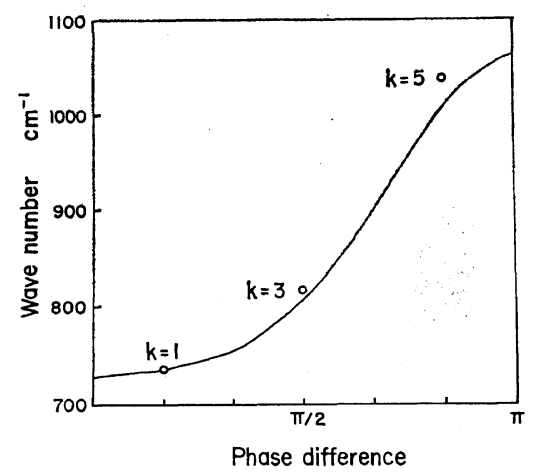

Figure 10. Observed frequencies and phase differences of the $r\left(\mathrm{CH}_{2}\right)$ modes of poly(pentamethylene sulfide). The solid line represents the $\mathrm{CH}_{2}$ rocking $\left(\nu_{8}\right)$ dispersion curve calculated for polymethylene chain by Tasumi, et al. (ref 9).
Table V. Correlation diagram for molecular, site, and space groups for poly(pentamethylene sulfide) crystal

\begin{tabular}{|c|c|c|}
\hline$\underset{\mathrm{C}_{2 \nabla}}{\text { Molecular group }}{ }^{\mathrm{a}}$ & Site group & $\begin{array}{l}\text { Space } \text { group }^{\mathrm{b}} \\
\mathrm{C}_{2 \mathrm{~h}}\end{array}$ \\
\hline $\begin{array}{l}\mathrm{A}_{1} \\
\mathrm{~A}_{2} \\
\mathrm{~B}_{1} \\
\mathrm{~B}_{2}\end{array}$ & $\equiv \mathrm{A}$ & $\begin{array}{l}\mathrm{A}_{\mathrm{g}} \\
\mathrm{B}_{\mathrm{g}} \\
\mathrm{A}_{\mathrm{u}} \\
\mathbf{B}_{\mathrm{u}}\end{array}$ \\
\hline
\end{tabular}

a $A_{1}(\perp), B_{1}(\|)$, and $B_{2}(\perp)$ species are infrared-active. b $A_{u}(\perp)$ and $B_{u}(\perp, \|)$ species are infrared active.

and the site group of the molecule in the crystal lattice is $C_{1}$. Hence one obtained the correlation among the molecular, site, and space groups. The correlation diagram given in Table $\mathrm{V}$ indicates that a molecular mode generates four modes in the crystal-field $\left(A_{g}, B_{g}, A_{u}\right.$, and $\left.B_{u}\right)$, and two of those are infrared-active $\left(A_{u}\right.$ and $\left.B_{u}\right)$. This is reflected in the observed vibrational spectra as band splitting. The infrared-active $r\left(\mathrm{CH}_{2}\right)$ modes $\left(\mathrm{B}_{2}\right)$ of a poly(pentamethylene sulfide) molecule should split into doublets. Actually, the band splitting is ob-

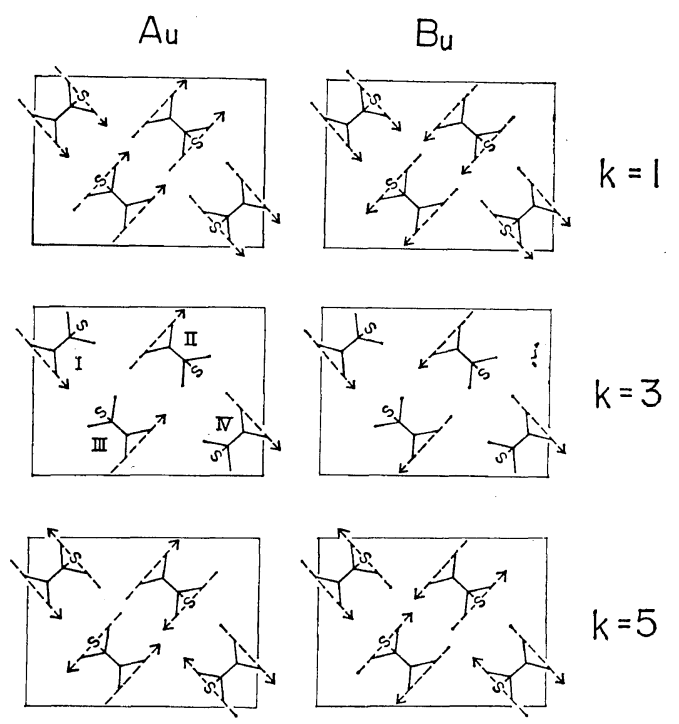

Figure 11. Schematic representations of the $r\left(\mathrm{CH}_{2}\right)$ modes belonging to the $A_{u}$ and $B_{u}$ species in the crystal lattice of poly(pentamethylene sulfide). The arrows (broken line) indicate the direction of the total transition moments associated with the $\mathrm{CH}_{2}$ groups in each side of the zigzag chain. 
served at about $727(k=1)$ and $1041 \mathrm{~cm}^{-1}(k=5)$ as seen in Figure 3.

Why only the band splitting of the $r\left(\mathrm{CH}_{2}\right)$ mode with $k=3$ (about $827 \mathrm{~cm}^{-1}$ ) is not observed can be understood by considering both the vibrational form and the crystal structure. The $r\left(\mathrm{CH}_{2}\right)$ modes belonging to the $\mathrm{A}_{\mathrm{u}}$ and $\mathrm{B}_{\mathrm{u}}$ species in the crystal lattice are schematically shown for each value of $k=1,3$, and 5 in Figure 11, in which the arrows (broken line) indicate the directions of the total transition moments associated with the $\mathrm{CH}_{2}$ groups on each side of the zigzag chain. The chains II, III, and IV are related to the chain I by the two-fold screw axis, the glide plane, and the inversion in the lattice, respectively (see Figure 4). For consideration of the crystal-field splitting of the infrared-active $r\left(\mathrm{CH}_{2}\right)$ mode, it is sufficient to pay attention to the interaction between the two molecular chains related by the two-fold screw axis in the crystal lattice (for example, the chains I and II), since the $r\left(\mathrm{CH}_{2}\right)$ modes belonging to the $A_{u}$ and $B_{u}$ species are symmetric and antisymmetric to the two-fold screw axis, respectively.

Whether the band splittings in the crystal-field take place or not, generally depends on the difference in the intermolecular interaction between two lattice modes ( $\mathbf{A}_{\mathrm{u}}$ and $\mathbf{B}_{\mathrm{u}}$ modes) under consideration. In the case of the rocking vibration with $k=3$, the hydrogen atoms of the methylene groups on the side of the sulfur atoms (this side is here named the S-side and another the $\mathrm{C}$-side) in each zigzag chain make no appreciable displacements as shown in Figures 9 and 11. For the effective interactions in the crystal, the methylene groups on the C-side of the chain II are sufficiently close to those on the S-side of the chain I (the interchain hydrogen-hydrogen distances, about $2.5 \AA$ ), but too far away from those on the $\mathrm{C}$-side of the chain I (the $\mathrm{H} \cdots \mathrm{H}$ distances, about $4.0 \AA$ ), as can be seen in Figure 11. It can be presumed from the vibrational feature and the molecular arrangement in the crystal lattice that the intermolecular interaction between the chains $I$ and II in the $\mathrm{A}_{\mathrm{u}}$ mode with $k=3$ may not be different from that in the $B_{u}$ mode. It may be due to the indistinguishable intermolecular interactions in the two lattice modes that the band splitting of the $r\left(\mathrm{CH}_{2}\right)$ mode with $k=3$ is not observed at room temperature. However one might expect to observe the band splitting at low temperatures, and this is confirmed in

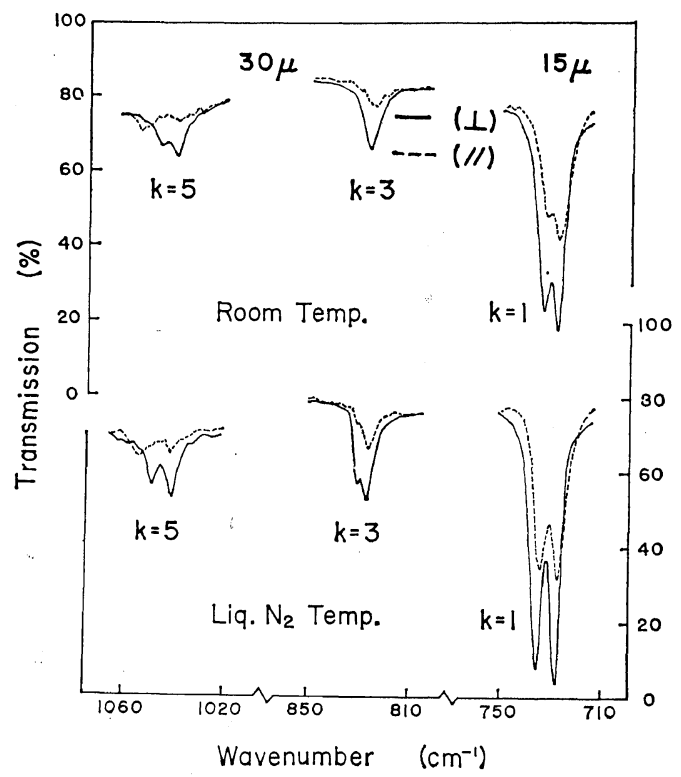

Figure 12. Spectral bands assigned to the $r\left(\mathrm{CH}_{2}\right)$ modes of poly(pentamethylene sulfide) at room temperature (upper part) and the liquid-nitrogen temperature (lower part).

Table VI. Observed values of the splitting of $\mathrm{CH}_{2}$ rocking modes for poly(pentamethylene sulfide) $\left(\mathrm{cm}^{-1}\right)$

\begin{tabular}{ccc}
\hline$k$-value & Room temp & Liquid $\mathrm{N}_{2}$ temp \\
\hline 5 & $\left\{\begin{array}{l}1047.5 \\
1041.0\end{array}(6.5)^{\mathrm{a}}\right.$ & $\left\{\begin{array}{l}1049.5 \\
1042.0\end{array}(7.5)\right.$ \\
3 & $\left\{\begin{array}{l}829.5 \\
827.0\end{array}(2.5)\right.$ \\
1 & $\left\{\begin{array}{l}732.5 \\
727.5\end{array}(5.0)\right.$ & $\left\{\begin{array}{l}735.0 \\
727.5\end{array}(7.5)\right.$
\end{tabular}

Polyethylene
$k=0$$\quad\left\{\begin{array}{l}731.0 \\ 720.0\end{array}(11.0) \quad\left\{\begin{array}{l}732.0 \\ 719.1\end{array}(12.9)^{\mathrm{b}}\right.\right.$.

a The values in parentheses indicate the splitting width $\Delta \nu$.

b Ref 19. 
fact by the infrared measurements at liquidnitrogen temperature.

When the specimen of poly(pentamethylene sulfide) is cooled from the room temperature to the liquid-nitrogen temperature, the singlet at $827 \mathrm{~cm}^{-1}(k=3)$ splits slightly into a doublet with a width of $2.5 \mathrm{~cm}^{-1}$, and then the splitting width $\Delta \nu$ for the doublets at about $727(k=1)$ and $1041 \mathrm{~cm}^{-1}(k=5)$ increases from 5.0 to 7.5 $\mathrm{cm}^{-1}$ and 6.5 to $7.5 \mathrm{~cm}^{-1}$, respectively, as shown in Figure 12 and Table VI. The similar temperature dependence of $\Delta \nu$ has been found for the correlation splitting of the $r\left(\mathrm{CH}_{2}\right)$ mode of polyethylene crystal. ${ }^{19,20}$ The doublet at about $1041 \mathrm{~cm}^{-1}$ does not show much of a temperature dependence in its splitting width, but the bands become much sharper as the temperature is lowered.

The magnitudes of the splittings are closely related to the interchain hydrogen-hydrogen $(\mathrm{H} \cdot \mathrm{.H})$ distances, and rather short $\mathrm{H} \cdot \mathrm{HH}$ distances may be required for the appearance of the doublet with appreciable splitting width. In the case of polyethylene, for example, the $\mathrm{H} \cdot \mathrm{HH}$ distances in the range of $2.5-2.9 \AA$ give rise to the doublet with $\Delta \nu=10 \mathrm{~cm}^{-1}$ for the $r\left(\mathrm{CH}_{2}\right)$ mode. ${ }^{21}$ The result of the X-ray analysis of poly(pentaethylene sulfide) indicates that some of the methylene groups have the $\mathrm{H}$...H distances in the range of 2.4-3.0 $\AA$. Nevertheless, the splitting widths are much smaller than those of polyethylene (about half). This fact may be explained by the aforementioned vibrational phase differences among the methylene groups in the poly(pentamethylene sulfide) molecule due to the existence of the sulfur atoms. On the contrary, the infraredactive $r\left(\mathrm{CH}_{2}\right)$ mode is in-phase $(\delta=0)$ in the polyethylene molecule.

Acknowledgement. The authors are much indebted to Mr. Yasuhiro Takahashi and Mr. Daisuke Makino* of this laboratory for their helpful advice and valuable discussion.

* Present address: Hitachi Chemical Industry Co., Ltd., Hitachi, Ibaragi, Japan.

\section{REFERENCES}

1. H. Tadokoro, "Structure of Crystalline Polyethers", Macromolecular Reviews, chapter 4, 119, 1967 and the references therein.

2. K. Imada, T. Miyakawa, Y. Chatani, H. Tadokoro, and S. Murahashi, Makromol. Chem., 83, 113 (1965).

3. T. Uchida and H. Tadokoro, J. Polym, Sci., Part A-2, 5, 63 (1967).

4. H. Tadokoro, Y. Takahashi, Y. Chatani, and H. Kakida, Makromol. Chem., 109, 96 (1967).

5. S. Kobayashi, H. Tadokoro, and Y. Chatani, ibid., 112, 225 (1968).

6. D. Makino, M. Kobayashi, and H. Tadokoro, J. Chem. Phys., 51, 3901 (1969).

7. H. Kakida, D. Makino, Y. Chatani, M. Kobayashi, and H. Tadokoro, Macromolecules, 3, 569 (1970).

8. G. Carazzolo and G. Valle, Makromol. Chem., 90, 66 (1966).

9. Y. Takahashi, H. Tadokoro, and Y. Chatani, J. Macromol. Sci., B2, 361 (1968).

10. H. Sakakihara, Y. Takahashi, and H. Tadokoro, Abstracts, SPSJ 18-th Annual Meeting, Tokyo, November 11, 1969, p407.

11. C. S. Mavel and A. Kotch, J. Amer. Chem. Soc., 73, 481 (1951).

12. H. J. M. Bowen, et al., "Tables of Interatomic Distances and Configurations in Molecules and Ions', The Chemical Society, London, 1958.

13. S. Arnott and A. J. Wonacott, Polymer, 7, 157 (1966).

14. Y. Takahashi, T. Satoh, and H. Tadokoro, J. Polym. Sci., Part A-2, in press.

15. P. W. Teare, Acta Cryst. allogr., 12, 294 (1959).

16. S. Kobayashi, H. Tadokoro, and Y. Chatani, unpublished.

17. See, for example, ref. 18 or R. G. Synder and J. H. Schachatschneider, Spectrochim. Acta, 19, 85 (1963).

18. M. Tasumi, T. Shimanouchi, and T. Miyazawa, J. Mol. Spectrosc., 9, 261 (1963).

19. G. W. King, R. M. Hainer, and M. O. McMahon, J. Appl. Phys., 20, 539 (1949).

20. R. G. Synder, J. Mol. Spectrosc., 7, 116 (1961).

21. M. Tasumi and T. Shimanouchi, J. Chem. Phys., 43, 1245 (1965). 